\title{
Single-Machine Scheduling with Accelerating Learning Effects
}

\author{
T. C. E. Cheng, ${ }^{1}$ Shih-Chang Tseng, ${ }^{2}$ Peng-Jen Lai, ${ }^{3}$ and Wen-Chiung Lee ${ }^{4}$ \\ ${ }^{1}$ Department of Logistics and Maritime Studies, The Hong Kong Polytechnic University, Hung Hom, Kowloon, Hong Kong \\ ${ }^{2}$ Department of Business Administration, National Central University, Taoyuan, Taiwan \\ ${ }^{3}$ Department of Mathematics, National Kaohsiung Normal University, Kaohsiung, Taiwan \\ ${ }^{4}$ Department of Statistics, Feng Chia University, Taichung, Taiwan
}

Correspondence should be addressed to Wen-Chiung Lee; wclee@fcu.edu.tw

Received 5 September 2013; Revised 16 October 2013; Accepted 16 October 2013

Academic Editor: Yunqiang Yin

Copyright (C) 2013 T. C. E. Cheng et al. This is an open access article distributed under the Creative Commons Attribution License, which permits unrestricted use, distribution, and reproduction in any medium, provided the original work is properly cited.

Scheduling with learning effects has been widely studied. However, there are situations where the learning effect might accelerate. In this paper, we propose a new model where the learning effect accelerates as time goes by. We derive the optimal solutions for the single-machine problems to minimize the makespan, total completion time, total weighted completion time, maximum lateness, maximum tardiness, and total tardiness.

\section{Introduction}

In classical scheduling, the processing times of the jobs are assumed to be constant over the entire planning horizon [1]. However, this assumption may not be appropriate for all the cases [2-5]. In many realistic situations, because the employees do the same job repeatedly, they learn how to do similar jobs more efficiently. As a result, the actual processing time of a job is shorter if it is scheduled later, rather than earlier in the sequence. This phenomenon is known as the "learning effect" and has been applied in scheduling problems. Dondeti and Mohanty [6], Biskup [7], Cheng and Wang [8], and Gawiejnowicz [9] are among the pioneers that incorporate the learning effect into scheduling problems. Dondeti and Mohanty [6] consider a single-machine scheduling problem in which the machine experiences learning as it continues to work. Biskup [7] points out that the learning effect in scheduling may arise in firms that produce similar jobs. Cheng and Wang [8] study a single-machine scheduling problem in which the job processing times will decrease as a result of learning. Since then, a number of researchers have considered the effects of learning in different scheduling settings, including single-machine scheduling, two-machine flowshop scheduling, and parallel machine scheduling. Cheng et al. [10], Gawiejnowicz [11], Biskup [12], and Rustogi and Strusevich [13] provide comprehensive reviews of scheduling models with time-dependent processing times.

Recently, Janiak and Rudek [14] introduce a new learning effect model into the scheduling field where learning is generalized in two ways. First, they allow each job to have a different learning effect to the operator. Second, they assume that the job processing time is a nonincreasing $k$-stepwise function, which in general is not restricted to a certain learning curve; hence, it can accurately fit every possible shape of a learning function. Wang et al. [15] consider some single-machine problems with a time-dependent learning effect. They find the optimal solutions or derive the worstcase error bounds for some proposed heuristic algorithms. Janiak and Rudek [16] consider and analyze a learning effect model in which the learning curve is S-shaped. They provide the NP-hard proofs for two cases of the problem to minimize the makespan. Lee and $\mathrm{Wu}$ [17] observe that robots with neural networks are used in computers, motor vehicles, and many assembly lines. The actions of a robot are constantly modified through self-learning in processing jobs. On the other hand, the operators in the control centre learn how to give commands efficiently through work experience. This motivates the consideration of both "position-based" and "sum-of-processing-time-based" learning effects simultaneously. Combining the models of Biskup [7] and Koulamas and Kyparisis [18], Cheng et al. [19] introduce a model with 
position-based and sum-of-processing-timed-based learning effects in which the actual processing time of a job is a function of the total normal processing times of the jobs already processed and of the job position in a schedule. $\mathrm{Wu}$ and Lee [20] study the problem with learning effects to minimize the total completion time in a multiple machine permutation flowshop. Janiak and Rudek [21] suggest a new approach, called multiability learning, which generalizes the existing ones and models more precisely real-life settings. They focus on the problem with the proposed learning model to minimize the makespan and provide optimal polynomial time algorithms for some special cases. Cheng et al. [22] develop a learning effect model where the actual job processing time is a logarithm function of the normal processing time of jobs already processed. They provide the optimal solutions for some single-machine problems. Lee et al. [23] consider a single-machine bi-criterion problem with learning effects and job release times. Wang et al. [24] study some single-machine problems with past-sequencedependent setup times. Lee et al. [25] investigate a singlemachine problem with the learning effect and release times to minimize the makespan. Ji and Cheng [26] consider a scheduling problem with job-dependent learning effects and multiple rate-modifying activities. They show that the problem to minimize the total completion time remains polynomially solvable. Wu and Lee [27] and Yin et al. [28] further generalize the model proposed by Cheng et al. [19]. Zhang and Yan [29] modify the models of Cheng et al. [19] and $\mathrm{Wu}$ and Lee [27] and provide the optimal solutions for some single-machine and flowshop problems. Wang et al. [30] and Wang and Wang [31] provide the optimal solutions for some single-machine problems with an exponential sum-ofactual-processing-time-based learning effect. Okołowski and Gawiejnowicz [32] consider a parallel-machine scheduling problem with DeJong's learning effect. For the makespan problem, they propose a sequential branch-and-bound algorithm and a parallel branch-and-bound algorithm. Wang et al. [33] consider resource allocation scheduling with learning effects where the processing time of a job is a function of the job's position in a sequence and its resource allocation. They provide a polynomial algorithm to find the optimal job sequence and resource allocation. Hsu et al. [34] and Kuo et al. [35] study the total completion time problem on unrelated parallel machines with past-sequence-dependent setup times and learning effects. Zhu et al. [36] investigate some singlemachine group scheduling problems with resource allocation and learning effects. Rudek [37] provide the computational complexity and solution algorithms for flowshop scheduling problems with the learning effect. Lai and Lee [38] propose a learning effect model where the actual processing time of a job is a general function of the normal processing times of the jobs already processed and the job's own scheduled position.

Biskup [12] classifies the existing models into two different approaches, namely, position-based learning effect and sum-of-processing-time-based learning effect models. He points out that position-based learning corresponds to situations where learning arises from processing-time independent operations like setting up machines. This is a realistic assumption for the case because the actual processing times of the jobs are mainly machine-driven. The sum-of-processingtime-based approach takes into account the experience that workers gain from producing the jobs. This might, for example, be the case for offset printing, where running the press itself is a highly complicated and error-prone process. In some precision manufacturing environments, such as the aircraft manufacturing industry, new employees must spend long time to become skilled workers. In this case, the learning effect is not evident in the early stage because of the low learning rate at that stage, but the learning rate will gradually increase as employees become skilled. In other words, the learning rate accelerates over time. This phenomenon also exists in labour-intensive industries, such as hand-made umbrellas, fans, and embroidery, in which craftsmen need to learn and perfect their crafts over a long period of time practising their skills. In this study we propose a scheduling model with the learning effect that might accelerate.

The remainder of this paper is organized as follows. We present the solution procedures for the single-machine problems to minimize the makespan, total completion time, total weighted completion time, maximum lateness, maximum tardiness, and total tardiness in Section 2. We conclude the paper in Section 3.

\section{Some Single-Machine Problems}

There are $n$ jobs ready to be processed on a single machine. For each job $j$, there is a normal processing time $p_{j}$, a due date $d_{j}$, and a weight $w_{j}$. Due to the learning effect as in [39], the actual processing time of job $j$ if it is scheduled in the $r$ th position of a sequence is

$$
p_{j[r]}=p_{j}\left(1+\sum_{k=1}^{r-1} \alpha_{r, k} p_{[k]}\right)^{a}
$$

for $r=1,2, \ldots, n$, where $a<0, p_{[k]}$ denotes the normal processing time of the job scheduled in the $k$ th position of the sequence and $\alpha_{r, i} \leq \alpha_{r, j}$ if $i<j$ and $\alpha_{r, j} \geq \alpha_{r^{\prime}, j} \geq 0$ if $r>r^{\prime}$. Throughout the paper, let $C_{j}, L_{j}=C_{j}-d_{j}$, and $T_{j}=\max \left\{0, C_{j}-d_{j}\right\}$ denote the completion time, lateness, and tardiness of job $j$, respectively. In addition, we let $C_{\text {max }}=\max \left\{C_{j}\right\}, L_{\max }=\max \left\{L_{j}\right\}$, and $T_{\max }=\max \left\{T_{j}\right\}$. Using the well-known three-field notation for describing scheduling problems, we denote the problems under study as $1\left|p_{j[r]}=p_{j}\left(1+\sum_{k=1}^{r-1} \alpha_{r, k} p_{[k]}\right)^{a}\right| C_{\max }, 1 \mid p_{j[r]}=p_{j}(1+$ $\left.\sum_{k=1}^{r-1} \alpha_{r, k} p_{[k]}\right)^{a}\left|\sum C_{i}, 1\right| p_{j[r]}=p_{j}\left(1+\sum_{k=1}^{r-1} \alpha_{r, k} p_{[k]}\right)^{a} \mid \sum w_{i} C_{i}$, $1\left|p_{j[r]}=p_{j}\left(1+\sum_{k=1}^{r-1} \alpha_{r, k} p_{[k]}\right)^{a}\right| L_{\max }, 1 \mid p_{j[r]}=p_{j}(1+$ $\left.\sum_{k=1}^{r-1} \alpha_{r, k} p_{[k]}\right)^{a} \mid T_{\text {max }}$, and $1\left|p_{j[r]}=p_{j}\left(1+\sum_{k=1}^{r-1} \alpha_{r, k} p_{[k]}\right)^{a}\right| \sum T_{i}$, respectively.

Before presenting the main results, we first state some lemmas that will be used in the proofs in the sequel.

Lemma 1. $(\theta-1)(1+c+u)^{a}-\theta(1+u+\lambda t)^{a}+(1+u+\lambda \theta t)^{a} \geq 0$ for $a<0, \theta \geq 1, \lambda \geq 0, c<0, t \geq 0, u>0$, and $1+c+u \geq 0$. 
Proof. Let $F(t)=(\theta-1)(1+c+u)^{a}-\theta(1+u+\lambda t)^{a}+$ $(1+u+\lambda \theta t)^{a}$. Taking the first derivative of $F(t)$ with respect to $t$, we have

$$
F^{\prime}(t)=a \lambda \theta\left\{(1+u+\lambda \theta t)^{a-1}-(1+u+\lambda t)^{a-1}\right\} \geq 0 .
$$

Since $a<0$ and $\theta \geq 1, F(t)$ is a nondecreasing function so $F(t) \geq F(0) \geq 0$. This completes the proof.

Lemma 2. $(1+u+c)^{a}(1-\theta)+\lambda_{2} \theta(1+u+\lambda t)^{a}-\lambda_{1}(1+$ $u+\lambda \theta t)^{a} \leq 0$ for $a<0, \theta \geq 1, \lambda \geq 0, c<0, t \geq 0,0 \leq \lambda_{2} \leq$ $\lambda_{1} \leq 1, u>0$, and $1+c+u \geq 0$.

Proof. Let $F(\theta)=(\theta-1)(1+u+c)^{a}+\lambda_{1}(1+u+\theta t)^{a}-\lambda_{2} \theta(1+$ $u+t)^{a}$. To show that $F(\theta)$ is nonnegative, it suffices to show that $F^{\prime}(\theta) \geq 0$ because $F(1)=(1+u+t)^{a}\left(\lambda_{1}-\lambda_{2}\right) \geq 0$. Taking the first derivative of $F(\theta)$ with respect to $\theta$, we have

$$
\begin{aligned}
F^{\prime}(\theta)= & (1+u+c)^{a}+a \lambda_{1} t(1+u+\theta t)^{a-1} \\
& -\lambda_{2}(1+u+t)^{a}
\end{aligned}
$$

Since $a<0, u>0, t \geq 0$, and $\lambda_{1} \geq 0$, we have

$$
\begin{aligned}
F^{\prime}(\theta) \geq & (1+u+c)^{a}+a \lambda_{1} t(1+u+t)^{a-1} \\
& -\lambda_{2}(1+u+t)^{a}=F^{\prime}(1) .
\end{aligned}
$$

To show that $F^{\prime}(1) \geq 0$, we define the following function:

$$
G(\xi, \eta)=(1+u+c)^{a}+a t \eta(1+u+t)^{a-1}-\xi(1+u+t)^{a} .
$$

First, we claim that $G(1,1) \geq 0$. We have

$$
\begin{aligned}
G(1,1)= & (1+u+c)^{a}+a t(1+u+t)^{a-1}-(1+u+t)^{a} \\
= & (1+u+c)^{a}-(1+u)^{a}+(1+u)^{a} \\
& -(1+u+t)^{a}+a t(1+u+t)^{a-1} .
\end{aligned}
$$

By the mean value theorem, there exists a $\sigma$ with $0 \leq \sigma \leq 1$ such that

$$
(1+u)^{a}-(1+u+t)^{a}=-\operatorname{ta}(1+u+\sigma t)^{a-1} .
$$

It follows that

$$
\begin{aligned}
G(1,1)= & (1+u+c)^{a}-(1+u)^{a}-t a(1+u+\sigma t)^{a-1} \\
& +a t(1+u+t)^{a-1} .
\end{aligned}
$$

Since $a<0$, we have $(1+u+c)^{a} \geq(1+u)^{a}$ and $(1+u+$ $\sigma t)^{a-1} \geq(1+u+t)^{a-1}$ for $0 \leq \sigma \leq 1$. Thus, $G(1,1) \geq 0$ and the claim is proved.

Since $G(\xi, \eta)$ is nonincreasing with respect to $\xi$ and $\eta$, we have $G(\xi, \eta) \geq G(1,1) \geq 0$. This implies that $F^{\prime}(1)=$ $G\left(\lambda_{2}, \lambda_{1}\right)$ is nonnegative. Thus, we have $F^{\prime}(\theta) \geq F^{\prime}(1) \geq 0$ and the proof is completed.

We prove the following properties using the pairwise job interchange technique. Suppose that $S$ and $S^{\prime}$ are two job schedules and the difference between $S$ and $S^{\prime}$ is a pairwise interchange of two adjacent jobs $i$ and $j$. That is, $S=$ $\left(\pi, i, j, \pi^{\prime}\right)$ and $S^{\prime}=\left(\pi, j, i, \pi^{\prime}\right)$, where $\pi$ and $\pi^{\prime}$ each denote a partial sequence. Furthermore, we assume that there are $r-1$ jobs in $\pi$. In addition, let $A$ denote the completion time of the last job in $\pi$. Under the proposed model, the completion times of jobs $i$ and $j$ in $S$ and $S^{\prime}$ are

$$
\begin{gathered}
C_{i}(S)=A+p_{i}\left(1+\sum_{k=1}^{r-1} \alpha_{r, k} p_{[k]}\right)^{a}, \\
C_{j}(S)=A+p_{i}\left(1+\sum_{k=1}^{r-1} \alpha_{r, k} p_{[k]}\right)^{a} \\
+p_{j}\left(1+\sum_{k=1}^{r-1} \alpha_{r+1, k} p_{[k]}+\alpha_{r+1, r} p_{i}\right)^{a}, \\
C_{j}\left(S^{\prime}\right)=A+p_{j}\left(1+\sum_{k=1}^{r-1} \alpha_{r, k} p_{[k]}\right)^{a}, \\
\left.C_{i}\left(S^{\prime}\right)=A+p_{j}\left(1+\sum_{k=1}^{r-1} \alpha_{r, k} p_{[k]}\right)^{a}\right)^{a} \\
+p_{i}\left(1+\sum_{k=1}^{r-1} \alpha_{r+1, k} p_{[k]}+\alpha_{r+1, r} p_{j}\right)^{\prime}
\end{gathered}
$$

Property 1. An optimal schedule for the $1 \mid p_{j[r]}=p_{j}(1+$ $\left.\sum_{k=1}^{r-1} \alpha_{r, k} p_{[k]}\right)^{a} \mid C_{\max }$ problem is obtained by sequencing the jobs in the shortest processing time (SPT) order.

Proof. Suppose that $p_{j} \geq p_{i}$. To show that $S$ dominates $S^{\prime}$, it suffices to show that $C_{j}(S) \leq C_{i}\left(S^{\prime}\right)$. Taking the difference between (10) and (12), we have

$$
\begin{aligned}
C_{i}\left(S^{\prime}\right)-C_{j}(S)= & \left(p_{j}-p_{i}\right)\left(1+\sum_{k=1}^{r-1} \alpha_{r, k} p_{[k]}\right)^{a} \\
& +p_{i}\left(1+\sum_{k=1}^{r-1} \alpha_{r+1, k} p_{[k]}+\alpha_{r+1, r} p_{j}\right)^{a} \\
& -p_{j}\left(1+\sum_{k=1}^{r-1} \alpha_{r+1, k} p_{[k]}+\alpha_{r+1, r} p_{i}\right)^{a} .
\end{aligned}
$$

Substituting $u=\sum_{k=1}^{r-1} \alpha_{r+1, k} p_{[k]}, \theta=p_{j} / p_{i}, c=\sum_{k=1}^{r-1}\left(\alpha_{r, k}-\right.$ $\left.\alpha_{r+1, k}\right) p_{[k]}, t=p_{i}$, and $\lambda=\alpha_{r+1, r}$ into (13), we have from Lemma 1 that $C_{j}\left(S^{\prime}\right) \geq C_{i}(S)$. This completes the proof.

Similar to the proof of Property 1, we have the following result.

Property 2. An optimal schedule for the problem $1 \mid p_{j[r]}=$ $p_{j}\left(1+\sum_{k=1}^{r-1} \alpha_{r, k} p_{[k]}\right)^{a} \mid \sum C_{i}$ is obtained by sequencing the jobs in the SPT order.

Rudek [40] shows that the total weighted completion time problem with sum-of-processing-time-based learning 
effects is at least NP-hard. Since it is a special case of our proposed model, the problem to minimize the total weighted completion time under the proposed model is at least NP-hard. Thus, the weighted shortest processing time first (WSPT) rule does not yield the optimal solution under the proposed model. However, we show in the next property that the WSPT rule provides an optimal solution for the total weighted completion time problem if the normal processing times and the weights are agreeable; that is, $p_{i} \leq p_{j}$ implies that $w_{i} \geq w_{j}$ for all jobs $i$ and $j$.

Property 3. An optimal schedule for the $1 \mid p_{j[r]}=p_{j}(1+$ $\left.\sum_{k=1}^{r-1} \alpha_{r, k} p_{[k]}\right)^{a} \mid \sum w_{i} C_{i}$ problem is obtained by sequencing the jobs in the WSPT order if the normal processing times and the weights are agreeable.

Proof. Suppose that $p_{i} \leq p_{j}$. From Property 1, we have $C_{j}(S) \leq C_{i}\left(S^{\prime}\right)$. Thus, to show that $S$ dominates $S^{\prime}$, it suffices to show that

$$
w_{i} C_{i}(S)+w_{j} C_{j}(S) \leq w_{j} C_{j}\left(S^{\prime}\right)+w_{i} C_{i}\left(S^{\prime}\right)
$$

From (9) to (12), we have

$$
\begin{aligned}
& w_{i} C_{i}(S)+w_{j} C_{j}(S)-\left[w_{j} C_{j}\left(S^{\prime}\right)+w_{i} C_{i}\left(S^{\prime}\right)\right] \\
& =w_{i}\left(A+p_{i}\left(1+\sum_{k=1}^{r-1} \alpha_{r, k} p_{[k]}\right)^{a}\right) \\
& -w_{j}\left(A+p_{j}\left(1+\sum_{k=1}^{r-1} \alpha_{r, k} p_{[k]}\right)^{a}\right) \\
& +w_{j}\left(A+p_{i}\left(1+\sum_{k=1}^{r-1} \alpha_{r, k} p_{[k]}\right)^{a}\right. \\
& \left.+p_{j}\left(1+\sum_{k=1}^{r-1} \alpha_{r+1, k} p_{[k]}+\alpha_{r+1, r} p_{i}\right)^{a}\right) \\
& -\left[w _ { i } \left(A+p_{j}\left(1+\sum_{k=1}^{r-1} \alpha_{r, k} p_{[k]}\right)^{a}\right.\right. \\
& \left.\left.+p_{i}\left(1+\sum_{k=1}^{r-1} \alpha_{r+1, k} p_{[k]}+\alpha_{r+1, r} p_{j}\right)^{a}\right)\right] \\
& =\left(w_{i}+w_{j}\right) p_{i}\left[\left(1+\sum_{k=1}^{r-1} \alpha_{r, k} p_{[k]}\right)^{a}\left(1-\frac{p_{j}}{p_{i}}\right)\right. \\
& +\frac{w_{j}}{w_{i}+w_{j}} \frac{p_{j}}{p_{i}} \\
& \times\left(1+\sum_{k=1}^{r-1} \alpha_{r+1, k} p_{[k]}+\alpha_{r+1, r} p_{i}\right)^{a}
\end{aligned}
$$

$$
\begin{aligned}
& -\frac{w_{i}}{w_{i}+w_{j}} \\
& \left.\times\left(1+\sum_{k=1}^{r-1} \alpha_{r+1, k} p_{[k]}+\alpha_{r+1, r} p_{j}\right)^{a}\right] .
\end{aligned}
$$

Substituting $\theta=p_{j} / p_{i}, \lambda_{1}=w_{i} /\left(w_{i}+w_{j}\right), \lambda_{2}=w_{j} /\left(w_{i}+w_{j}\right)$, $u=\sum_{k=1}^{r-1} \alpha_{r+1, k} p_{[k]}, t=p_{i}, c=\sum_{k=1}^{r-1}\left(\alpha_{r, k}-\alpha_{r+1, k}\right) p_{[k]}$, and $\lambda=\alpha_{r+1, r}$ into (15), we have

$$
\begin{gathered}
w_{i} C_{i}(S)+w_{j} C_{j}(S)-\left[w_{i} C_{i}\left(s^{\prime}\right)+w_{j} C_{j}\left(s^{\prime}\right)\right] \\
=\left(w_{i}+w_{j}\right) p_{i}[ \\
{[1+u+c)^{a}(1-\theta)} \\
+\lambda_{2} \theta(1+u+\lambda t)^{a} \\
\left.-\lambda_{1}(1+u+\lambda \theta t)^{a}\right] .
\end{gathered}
$$

From Lemma 2, we have $w_{i} C_{i}(S)+w_{j} C_{j}(S)-\left[w_{i} C_{i}\left(S^{\prime}\right)+\right.$ $\left.w_{j} C_{j}\left(S^{\prime}\right)\right] \leq 0$. Therefore, repeating this job interchange argument for all the jobs not sequenced in the WSPT order completes the proof for the $1\left|p_{j[r]}=p_{j}\left(1+\sum_{k=1}^{r-1} \alpha_{r, k} p_{[k]}\right)^{a}\right| \sum w_{i} C_{i}$ problem.

Next, we show that the earliest due date (EDD) rule yields an optimal solution under the proposed models if the normal processing times and due dates are agreeable; that is, $d_{i} \leq d_{j}$ implies that $p_{i} \leq p_{j}$ for all jobs $i$ and $j$.

Property 4. An optimal schedule for the $1 \mid p_{j[r]}=p_{j}(1+$ $\left.\sum_{k=1}^{r-1} \alpha_{r, k} p_{[k]}\right)^{a} \mid L_{\max }$ problem is obtained by sequencing the jobs in the EDD order if the normal processing times and due dates are agreeable.

Proof. Suppose that $d_{i} \leq d_{j}$, which implies that $p_{i} \leq p_{j}$. The maximum lateness among the first $r-1$ jobs is the same since they are processed in the same order. Since the makespan is minimized by the SPT rule (Property 1 ), the maximum lateness in the partial sequence $\pi^{\prime}$ of $S$ will not be greater than that in the partial sequence $\pi^{\prime}$ of $S^{\prime}$. Thus, to prove that the maximum lateness of $S$ is less than or equal to that of $S^{\prime}$, it suffices to show that $\max \left\{L_{i}(S), L_{j}(S)\right\} \leq L_{i}\left(S^{\prime}\right)$.

From (9) to (12), we can derive that the lateness of jobs $i$ and $j$ in $S$ is

$$
\begin{gathered}
L_{i}(S)=A+p_{i}\left(1+\sum_{k=1}^{r-1} \alpha_{r, k} p_{[k]}\right)^{a}-d_{i}, \\
L_{j}(S)=A+p_{i}\left(1+\sum_{k=1}^{r-1} \alpha_{r, k} p_{[k]}\right)^{a} \\
+p_{j}\left(1+\sum_{k=1}^{r-1} \alpha_{r+1, k} p_{[k]}+\alpha_{r+1, r} p_{i}\right)^{a}-d_{j} .
\end{gathered}
$$


Similarly, the lateness of jobs $j$ and $i$ in $S^{\prime}$ is

$$
\begin{aligned}
L_{j}\left(S^{\prime}\right)=A+p_{j}\left(1+\sum_{k=1}^{r-1} \alpha_{r, k} p_{[k]}\right)^{a}-d_{j} \\
L_{i}\left(S^{\prime}\right)=A+p_{j}\left(1+\sum_{k=1}^{r-1} \alpha_{r, k} p_{[k]}\right)^{a} \\
+p_{i}\left(1+\sum_{k=1}^{r-1} \alpha_{r, k} p_{[k]}+\alpha_{r+1, r} p_{j}\right)^{a}-d_{i} .
\end{aligned}
$$

Thus, $L_{i}(S) \leq L_{i}\left(S^{\prime}\right)$ since $p_{i} \leq p_{j}$ and $p_{i}\left(1+\sum_{k=1}^{r-1} \alpha_{r, k} p_{[k]}+\right.$ $\left.\alpha_{r+1, r} p_{j}\right)^{a} \geq 0$. From Property 1 and $d_{i} \leq d_{j}$, we see that $L_{j}(S) \leq L_{i}\left(S^{\prime}\right)$. Thus, $S$ dominates $S^{\prime}$. Therefore, repeating this job interchange argument for all the jobs not sequenced in the EDD order completes the proof of the result for the $1\left|p_{j[r]}=p_{j}\left(1+\sum_{k=1}^{r-1} \alpha_{r, k} p_{[k]}\right)^{a}\right| L_{\max }$ problem. result.

Similar to the proof of Property 4, we have the following

Property 5. An optimal schedule for the $1 \mid p_{j[r]}=p_{j}(1+$ $\left.\sum_{k=1}^{r-1} \alpha_{r, k} p_{[k]}\right)^{a} \mid T_{\max }$ problem is obtained by sequencing the jobs in the EDD order if the normal processing times and due dates are agreeable.

Property 6. An optimal schedule for the $1 \mid p_{j[r]}=p_{j}(1+$ $\left.\sum_{k=1}^{r-1} \alpha_{r, k} p_{[k]}\right)^{a} \mid \sum T_{i}$ problem is obtained by sequencing the jobs in the EDD order if the normal processing times and due dates are agreeable.

Proof. Suppose that $d_{i} \leq d_{j}$, which implies $p_{i} \leq p_{j}$. The total tardiness of the first $r-1$ jobs is the same since they are processed in the same order. Since the makespan is minimized by the SPT rule (Property 1 ), the total tardiness of the partial sequence $\pi^{\prime}$ in $S$ will not be greater than that of the partial sequence $\pi^{\prime}$ in $S^{\prime}$. Thus, to prove that the total tardiness of $S$ is less than or equal to that of $S^{\prime}$, it suffices to show that

$$
T_{i}(S)+T_{j}(S) \leq T_{j}\left(S^{\prime}\right)+T_{i}\left(S^{\prime}\right) .
$$

From (9) to (12), we can derive that the tardiness of jobs $i$ and $j$ in $S$ is

$$
\begin{gathered}
T_{i}(S)=\max \left\{A+p_{i}\left(1+\sum_{k=1}^{r-1} \alpha_{r, k} p_{[k]}\right)^{a}-d_{i}, 0\right\}, \\
T_{j}(S)=\max \left\{A+p_{i}\left(1+\sum_{k=1}^{r-1} \alpha_{r, k} p_{[k]}\right)^{a}\right. \\
\left.+p_{j}\left(1+\sum_{k=1}^{r-1} \alpha_{r+1, k} p_{[k]}+\alpha_{r+1, r} p_{i}\right)^{a}-d_{j}, 0\right\} .
\end{gathered}
$$

Similarly, the tardiness of jobs $j$ and $i$ in $S^{\prime}$ is

$$
\begin{aligned}
T_{j}\left(S^{\prime}\right)=\max \left\{A+p_{j}\left(1+\sum_{k=1}^{r-1} \alpha_{r, k} p_{[k]}\right)^{a}-d_{j}, 0\right\}, \\
T_{i}\left(S^{\prime}\right)=\max \left\{A+p_{j}\left(1+\sum_{k=1}^{r-1} \alpha_{r, k} p_{[k]}\right)^{a}\right. \\
\left.+p_{i}\left(1+\sum_{k=1}^{r-1} \alpha_{r, k} p_{[k]}+\alpha_{r+1, r} p_{j}\right)^{a}-d_{i}, 0\right\} .
\end{aligned}
$$

To compare the total tardiness of jobs $i$ and $j$ in $S$ and $S^{\prime}$, we consider two cases. In the first case where $A+p_{j}(1+$ $\left.\sum_{k=1}^{r-1} \alpha_{r, k} p_{[k]}\right)^{a} \leq d_{j}$, the total tardiness of jobs $i$ and $j$ in $S$ and in $S^{\prime}$ is

$T_{i}(S)+T_{j}(S)$

$$
\begin{aligned}
&=\max \{A\left.+p_{i}\left(1+\sum_{k=1}^{r-1} \alpha_{r, k} p_{[k]}\right)^{a}-d_{i}, 0\right\} \\
&+\max \left\{A+p_{i}\left(1+\sum_{k=1}^{r-1} \alpha_{r, k} p_{[k]}\right)^{a}\right. \\
&\left.+p_{j}\left(1+\sum_{k=1}^{r-1} \alpha_{r+1, k} p_{[k]}+\alpha_{r+1, r} p_{i}\right)^{a}-d_{j}, 0\right\},
\end{aligned}
$$

$T_{j}\left(S^{\prime}\right)+T_{i}\left(S^{\prime}\right)$

$$
\begin{aligned}
=\max \{A & +p_{j}\left(1+\sum_{k=1}^{r-1} \alpha_{r, k} p_{[k]}\right)^{a} \\
& \left.+p_{i}\left(1+\sum_{k=1}^{r-1} \alpha_{r, k} p_{[k]}+\alpha_{r+1, r} p_{j}\right)^{a}-d_{i}, 0\right\} .
\end{aligned}
$$

Suppose that neither $T_{i}(S)$ nor $T_{j}(S)$ is zero. Note that this is the most restrictive case since it comprises the case where either one or both $T_{i}(S)$ and $T_{j}(S)$ are zero. From Property 1 and $d_{i} \leq d_{j}$, we have

$$
\begin{aligned}
\left\{T_{j}\left(S^{\prime}\right)+T_{i}\left(S^{\prime}\right)\right\}-\left\{T_{i}(S)+T_{j}(S)\right\} & \left(p_{j}-p_{i}\right)\left(1+\sum_{k=1}^{r-1} \alpha_{r, k} p_{[k]}\right)^{a} \\
& +p_{i}\left(1+\sum_{k=1}^{r-1} \alpha_{r, k} p_{[k]}+\alpha_{r+1, r} p_{j}\right)^{a} \\
& -p_{j}\left(1+\sum_{k=1}^{r-1} \alpha_{r+1, k} p_{[k]}+\alpha_{r+1, r} p_{i}\right)^{a} \\
& +d_{j}-A-p_{i}\left(1+\sum_{k=1}^{r-1} \alpha_{r, k} p_{[k]}\right)^{a} \geq 0 .
\end{aligned}
$$


Thus, $\left\{T_{j}\left(S^{\prime}\right)+T_{i}\left(S^{\prime}\right)\right\}-\left\{T_{i}(S)+T_{j}(S)\right\} \geq 0$ in the first case. In the second case where $A+p_{j}\left(1+\sum_{k=1}^{r-1} \alpha_{r, k} p_{[k]}\right)^{a}>d_{j}$, the total tardiness of jobs $i$ and $j$ in $S$ and $S^{\prime}$ is

$$
\begin{aligned}
& T_{i}(S)+T_{j}(S) \\
& =\max \left\{A+p_{i}\left(1+\sum_{k=1}^{r-1} \alpha_{r, k} p_{[k]}\right)^{a}-d_{i}, 0\right\} \\
& \quad \times \max \left\{A+p_{i}\left(1+\sum_{k=1}^{r-1} \alpha_{r, k} p_{[k]}\right)^{a}\right. \\
& \left.+p_{j}\left(1+\sum_{k=1}^{r-1} \alpha_{r+1, k} p_{[k]}+\alpha_{r+1, r} p_{i}\right)^{a}-d_{j}, 0\right\} \\
& T_{j}\left(S^{\prime}\right)+T_{i}\left(S^{\prime}\right) \\
& =2 A+2 p_{j}\left(1+\sum_{k=1}^{r-1} \alpha_{r, k} p_{[k]}\right)^{a} \\
& +p_{i}\left(1+\sum_{k=1}^{r-1} \alpha_{r, k} p_{[k]}+\alpha_{r+1, r} p_{j}\right)^{a}-d_{i}-d_{j} .
\end{aligned}
$$

Suppose that neither $T_{i}(S)$ nor $T_{j}(S)$ is zero. From Property $1, d_{i} \leq d_{j}$ and $p_{i} \leq p_{j}$, we have

$$
\begin{aligned}
& \left\{T_{j}\left(S^{\prime}\right)+T_{i}\left(S^{\prime}\right)\right\}-\left\{T_{i}(S)+T_{j}(S)\right\} \\
& =2\left(p_{j}-p_{i}\right)\left(1+\sum_{k=1}^{r-1} \alpha_{r, k} p_{[k]}\right)^{a} \\
& \quad+p_{i}\left(1+\sum_{k=1}^{r-1} \alpha_{r, k} p_{[k]}+\alpha_{r+1, r} p_{j}\right)^{a} \\
& \quad-p_{j}\left(1+\sum_{k=1}^{r-1} \alpha_{r+1, k} p_{[k]}+\alpha_{r+1, r} p_{i}\right)^{a} \geq 0 .
\end{aligned}
$$

Thus, $\left\{T_{j}\left(S^{\prime}\right)+T_{i}\left(S^{\prime}\right)\right\}-\left\{T_{i}(S)+T_{j}(S)\right\} \geq 0$ in the second case. Thus, $S$ dominates $S^{\prime}$. Therefore, repeating this job interchange argument for all the jobs not sequenced in the EDD order completes the proof of the result for the $1 \mid p_{j[r]}=$ $p_{j}\left(1+\sum_{k=1}^{r-1} \alpha_{r, k} p_{[k]}\right)^{a} \mid \sum T_{i}$ problem.

\section{Conclusions}

In this paper, we propose a new scheduling model where the learning effect accelerates. We derive the optimal solutions for some single-machine problems. For the makespan and the total completion time problems, we show that the SPT rule yields the optimal schedules. For the total weighted completion time problem, we show that the WSPT rule yields the optimal schedule if the normal processing times and weights are agreeable. Furthermore, we prove that the EDD rule yields the optimal schedules for the maximum lateness, maximum tardiness, and the total tardiness problems if the normal processing times and due dates are agreeable.

\section{Acknowledgments}

The authors are grateful to the referees for their comments. This work is supported by the National Science Council of Taiwan, under Grant no. NSC 1000-2221-E-035-029-MY3.

\section{References}

[1] M. Pinedo, Scheduling: Theory, Algorithms, and Systems, Third Edition, Prentice Hall, Upper Saddle River, NJ, USA, 2008.

[2] W. H. Wu, "A two-agent single-machine scheduling problem with learning and deteriorating considerations," Mathematical Problems in Engineering, vol. 2013, Article ID 648082, 18 pages, 2013.

[3] P. Liu and X. Tian, "Two-agent single-machine scheduling with resource-dependent starting times," Mathematical Problems in Engineering, vol. 2013, Article ID 805261, 5 pages, 2013.

[4] D. C. Li and P. H. Hsu, "Competitive two-agent scheduling with learning effect and release times on a single machine," Mathematical Problems in Engineering, vol. 2013, Article ID 754826, 9 pages, 2013.

[5] J. Y. Kung, Y. P. Chao, K. I. Lee, C. C. Kang, and W. C. Lin, “Twoagent single-machine scheduling of jobs with time-dependent processing times and ready times," Mathematical Problems in Engineering, vol. 2013, Article ID 806325, 13 pages, 2013.

[6] V. R. Dondeti and B. B. Mohanty, "Impact of learning and fatigue factors on single machine scheduling with penalties for tardy jobs," European Journal of Operational Research, vol. 105, no. 3, pp. 509-524, 1998.

[7] D. Biskup, "Single-machine scheduling with learning considerations," European Journal of Operational Research, vol. 115, no. 1, pp. 173-178, 1999.

[8] T. C. E. Cheng and G. Wang, "Single machine scheduling with learning effect considerations," Annals of Operations Research, vol. 98, no. 1-4, pp. 273-290, 2000.

[9] S. Gawiejnowicz, "A note on scheduling on a single processor with speed dependent on a number of executed jobs," Information Processing Letters, vol. 57, no. 6, pp. 297-300, 1996.

[10] T. C. E. Cheng, Q. Ding, and B. M. T. Lin, "A concise survey of scheduling with time-dependent processing times," European Journal of Operational Research, vol. 152, no. 1, pp. 1-13, 2004.

[11] S. Gawiejnowicz, Time-Dependent Scheduling, Springer, Berlin, Germany, 2008.

[12] D. Biskup, "A state-of-the-art review on scheduling with learning effects," European Journal of Operational Research, vol. 188, no. 2, pp. 315-329, 2008.

[13] K. Rustogi and V. A. Strusevich, "Simple matching vs linear assignment in scheduling models with positional effects: a critical review," European Journal of Operational Research, vol. 222, no. 3, pp. 393-407, 2012.

[14] A. Janiak and R. Rudek, "A new approach to the learning effect: beyond the learning curve restrictions," Computers and Operations Research, vol. 35, no. 11, pp. 3727-3736, 2008.

[15] J. B. Wang, C. T. Ng, T. C. E. Cheng, and L. L. Liu, "Singlemachine scheduling with a time-dependent learning effect," International Journal of Production Economics, vol. 111, no. 2, pp. 802-811, 2008. 
[16] A. Janiak and R. Rudek, "Experience-based approach to scheduling problems with the learning effect," IEEE Transactions on Systems, Man, and Cybernetics A, vol. 39, no. 2, pp. 344-357, 2009.

[17] W. C. Lee and C. C. Wu, "Some single-machine and m-machine flowshop scheduling problems with learning considerations," Information Sciences, vol. 179, no. 22, pp. 3885-3892, 2009.

[18] C. Koulamas and G. J. Kyparisis, "Single-machine and twomachine flowshop scheduling with general learning functions," European Journal of Operational Research, vol. 178, no. 2, pp. 402-407, 2007.

[19] T. C. E. Cheng, C. Wu, and W. Lee, "Some scheduling problems with sum-of-processing-times-based and job-position-based learning effects," Information Sciences, vol. 178, no. 11, pp. 24762487, 2008.

[20] C. C. Wu and W. C. Lee, "A note on the total completion time problem in a permutation flowshop with a learning effect," European Journal of Operational Research, vol. 192, no. 1, pp. 343-347, 2009.

[21] A. Janiak and R. Rudek, "A note on a makespan minimization problem with a multi-ability learning effect," Omega, vol. 38, no. 3-4, pp. 213-217, 2010.

[22] T. C. E. Cheng, P. Lai, C. Wu, and W. Lee, "Single-machine scheduling with sum-of-logarithm-processing-times-based learning considerations," Information Sciences, vol. 179, no. 18, pp. 3127-3135, 2009.

[23] W. C. Lee, C. C. Wu, and M. F. Liu, "A single-machine bicriterion learning scheduling problem with release times," Expert Systems with Applications, vol. 36, no. 7, pp. 10295-10303, 2009.

[24] J. B. Wang, D. Wang, L. Y. Wang, L. Lin, N. Yin, and W. Wang, "Single machine scheduling with exponential time-dependent learning effect and past-sequence-dependent setup times," Computers and Mathematics with Applications, vol. 57, no. 1, pp. 9-16, 2009.

[25] W. C. Lee, C. C. Wu, and P. H. Hsu, "A single-machine learning effect scheduling problem with release times," Omega, vol. 38, no. 1-2, pp. 3-11, 2010.

[26] M. Ji and T. C. E. Cheng, "Scheduling with job-dependent learning effects and multiple rate-modifying activities," Information Processing Letters, vol. 110, no. 11, pp. 460-463, 2010.

[27] C. C. Wu and W. C. Lee, "Single-machine and flowshop scheduling with a general learning effect model," Computers and Industrial Engineering, vol. 56, no. 4, pp. 1553-1558, 2009.

[28] Y. Q. Yin, D. H. Xu, K. B. Sun, and H. X. Li, "Some scheduling problems with general position-dependent and time-dependent learning effects," Information Sciences, vol. 179, no. 14, pp. 24162425, 2009 .

[29] X. G. Zhang and G. L. Yan, "Machine scheduling problems with a general learning effect," Mathematical and Computer Modelling, vol. 51, no. 1-2, pp. 84-90, 2010.

[30] J. B. Wang, L. H. Sun, and L. Y. Sun, "Scheduling jobs with an exponential sum-of-actual-processing-time-based learning effect," Computers and Mathematics with Applications, vol. 60, no. 9, pp. 2673-2678, 2010.

[31] J. B. Wang and J. J. Wang, "Single-machine scheduling jobs with exponential learning functions," Computers and Industrial Engineering, vol. 60, no. 4, pp. 755-759, 2011.

[32] D. Okołowski and S. Gawiejnowicz, "Exact and heuristic algorithms for parallel-machine scheduling with DeJong's learning effect," Computers and Industrial Engineering, vol. 59, no. 2, pp. 272-279, 2010
[33] D. Wang, M. Z. Wang, and J. B. Wang, "Single-machine scheduling with learning effect and resource-dependent processing times," Computers and Industrial Engineering, vol. 59, no. 3, pp. 458-462, 2010.

[34] C. J. Hsu, W. H. Kuo, and D. L. Yang, "Unrelated parallel machine scheduling with past-sequence-dependent setup time and learning effects," Applied Mathematical Modelling, vol. 35, no. 3, pp. 1492-1496, 2011

[35] W. H. Kuo, C. J. Hsu, and D. L. Yang, "Some unrelated parallel machine scheduling problems with past-sequence-dependent setup time and learning effects," Computers and Industrial Engineering, vol. 61, no. 1, pp. 179-183, 2011.

[36] Z. G. Zhu, L. Y. Sun, F. Chu, and M. Liu, "Single-machine group scheduling with resource allocation and learning effect," Computers and Industrial Engineering, vol. 60, no. 1, pp. 148-157, 2011.

[37] R. Rudek, "Computational complexity and solution algorithms for flowshop scheduling problems with the learning effect," Computers and Industrial Engineering, vol. 61, no. 1, pp. 20-31, 2011.

[38] P. J. Lai and W. C. Lee, "Single-machine scheduling with general sum-of-processing-time-based and position-based learning effects," Omega, vol. 39, no. 5, pp. 467-471, 2011.

[39] T. C. E. Cheng, S. C. Tseng, P. J. Lai, and W. C. Lee, "Singlemachine scheduling with accelerating deterioration effects," Optimization Letters, 2012.

[40] R. Rudek, "The single processor total weighted completion time scheduling problem with the sum-of-processing-time based learning model," Information Sciences, vol. 199, pp. 216-229, 2012. 


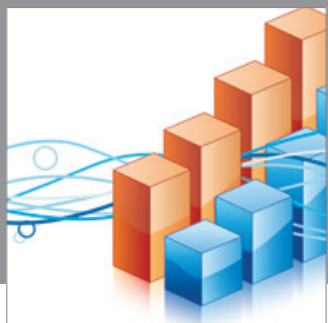

Advances in

Operations Research

mansans

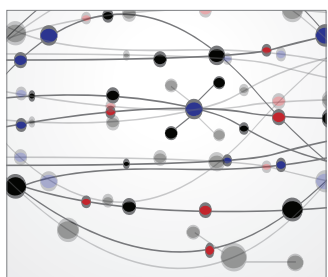

The Scientific World Journal
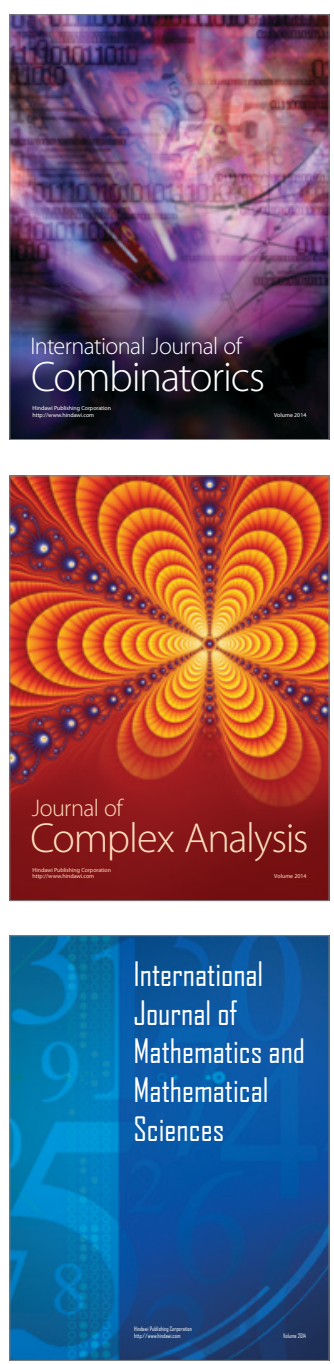
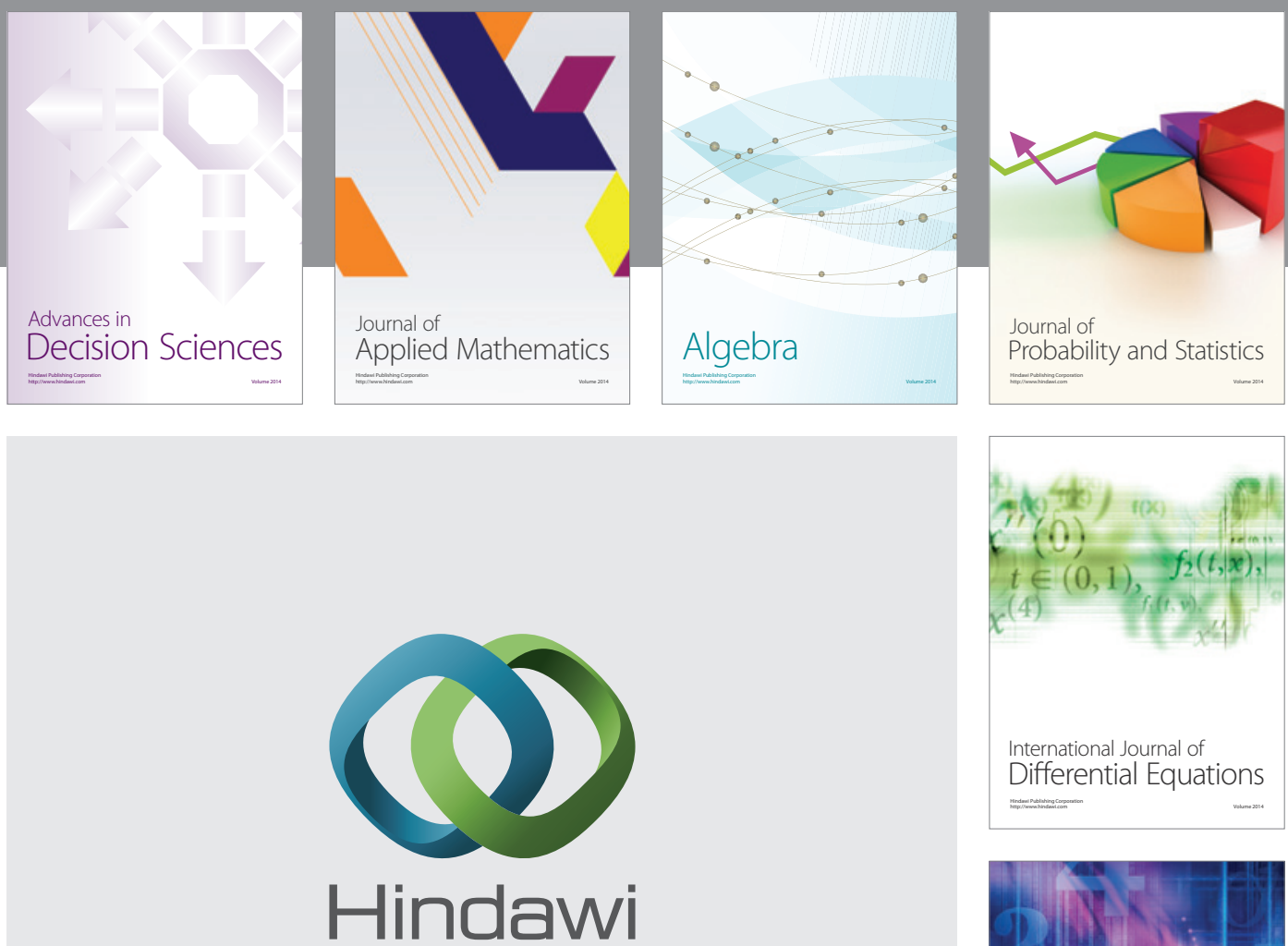

Submit your manuscripts at http://www.hindawi.com
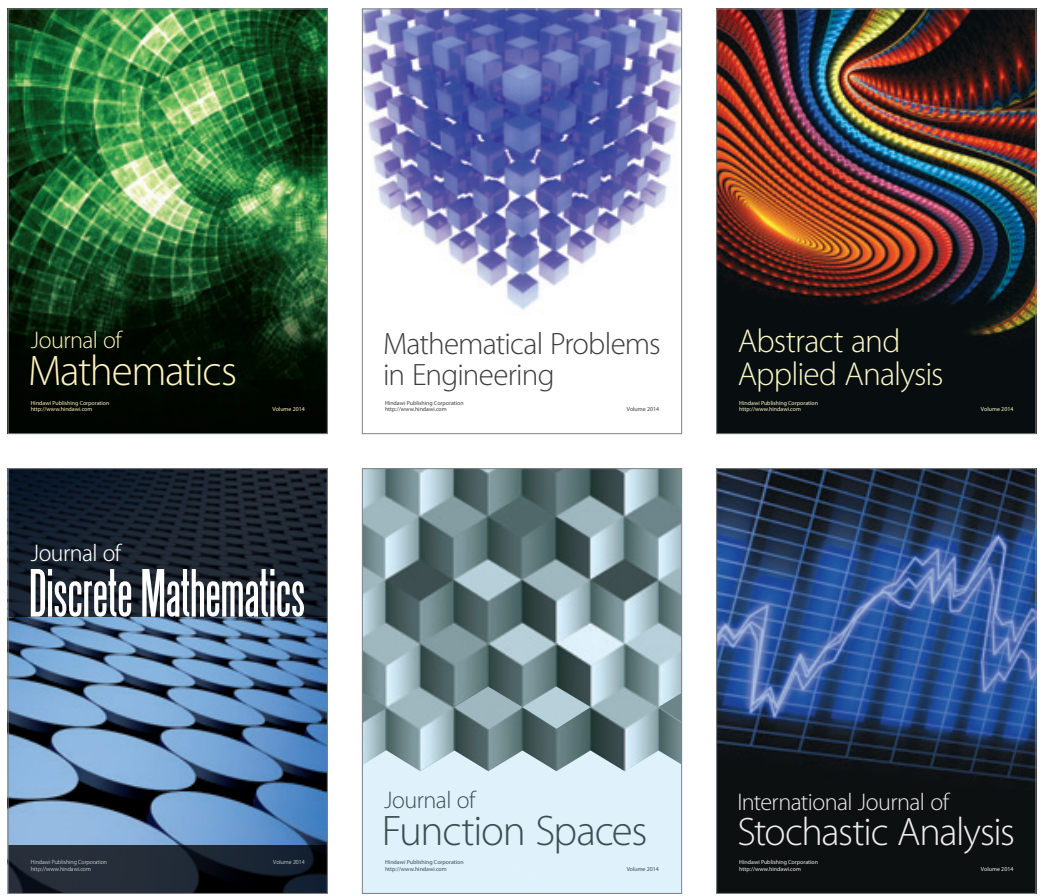

Journal of

Function Spaces

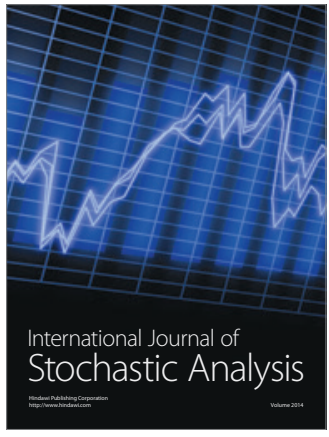

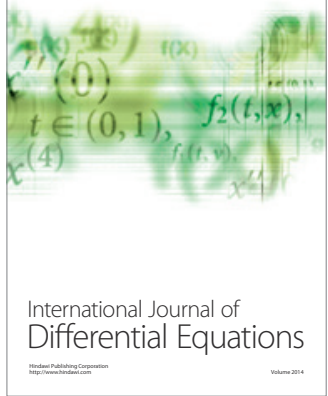
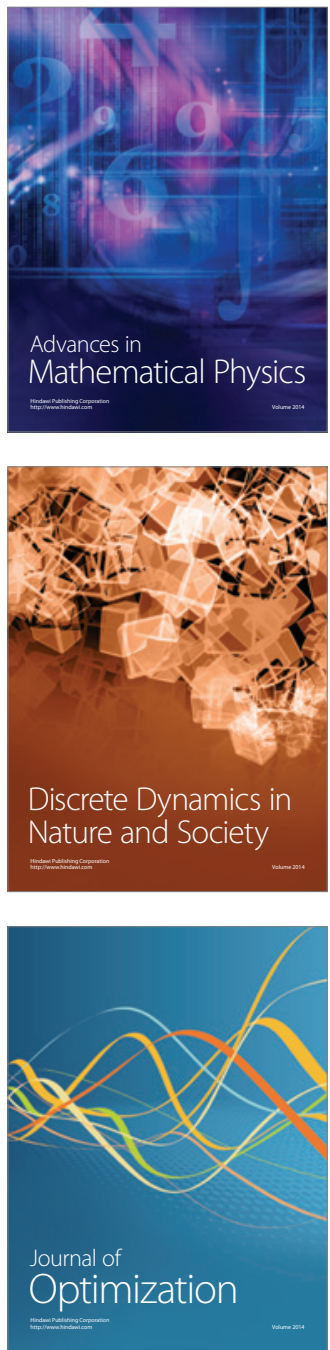